\title{
RELATIVISTIC EFFECTS ON PARALLEL WHISTLER-MODE PROPAGATION AND INSTABILITY
}

\author{
S. S. SAZHIN \\ Department of Physics, University of Sheffield, U.K. \\ and \\ N. M. TEMME \\ Centrum voor Wiskunde en Informatica, Amsterdam, The Netherlands
}

(Received 7 October, 1989)

\begin{abstract}
Relativistic effects on parallel whistler-mode propagation and instability are considered analytically in some limiting cases relevant to magnetospheric and/or astrophysical conditions. The corresponding wave dispersion equation for a weakly relativistic anisotropic plasma is expressed in terms of generalized Shkarofsky functions. Asymptotic presentation of these functions is found in the limit of large wave refractive indices. Based on this presentation, a new analytical expression for whistler-mode refractive index is obtained and analysed. It is pointed out that relativistic effects increase the value of anisotropy above which the waves are unstable, in agreement with the results of the earlier numerical analysis. This increase is particularly important for whistler-mode propagation in a rarefied, hot plasma but could be potentially observed in the magnetosphere of the Earth in the region outside the plasmasphere.
\end{abstract}

\section{Introduction}

Although the general dispersion equation describing wave propagation in a relativistic plasma is fairly well known (see, e.g., Trubnikov, 1959), its solution appears to be very complicated even for numerical methods. This equation can be simplified if we consider weakly relativistic limit, i.e., assume that the characteristic thermal velocity of charged particles is well below the velocity of light (see, e.g., Shkarofsky, 1966, 1986). Further simplification is possible if we restrict our analysis to particular types of waves. The waves which seem to be interesting both from the general theoretical point of view and from the point of view of their applications in magnetospheric and/or astrophysical conditions are parallel whistler-mode waves. The dispersion equation for these waves is particularly simple and convenient for analytical and numerical analysis. Observations of these waves in the magnetosphere of the Earth and their role in the dynamics of the magnetospheric plasma were discussed by many authors (see, e.g., Sazhin, 1982; Carpenter, 1988; Helliwell, 1988). These waves have also been observed in the magnetospheres of other planets (Anderson, 1983; Inan, 1987; Scarf et al., 1987).

The dispersion equation for parallel whistler-mode waves in a weakly relativistic plasma has been analysed numerically and analytically by Jacquinot and Leloup (1971), Tsang (1984), Robinson (1987a), Sazhin (1987a, b, 1989a), Bornatici and Ruffina (1989), and others. The main conclusion of these papers is that even in a weakly relativistic plasma the relativistic effects on whistler-mode propagation, instability and damping are not necessarily negligibly small. Hence, further analysis of these waves 
seems to be particularly interesting and important. This is done in the present paper.

In what follows we analyse analytically the parallel whistler-mode dispersion equation in a weakly relativistic plasma and obtain its asymptotic solution which is complementary to those obtained beforehand. In Section 2 this equation is presented in terms of generalized Shkarofsky functions. Some asymptotical properties of these functions are discussed in Section 3. The results of the latter section are applied to the analysis of whistler-mode propagation (Section 4) as well as to the analysis of marginal stability of these waves (Section 5). In the latter section we also discuss possible applications of our results to the conditions in the magnetosphere of the Earth. The main results of the paper are summarized in Section 6 .

\section{Dispersion Equation}

Similarly to Sazhin (1987a, b, 1989a) we assume the electron distribution function in the form:

$$
f\left(p_{\perp}, p_{\|}\right)=\left(j ! \pi^{3 / 2} p_{0 \perp}^{2 j+2} p_{0 \|}\right)^{-1} p_{\perp}^{2 j} \exp \left(-\frac{p_{\perp}^{2}}{p_{0 \perp}^{2}}-\frac{p_{\|}^{2}}{p_{0 \|}^{2}}\right),
$$

where $p_{0 \perp(\|)}$ is the electron thermal momentum in the direction perpendicular (parallel) to the magnetic field, $p_{\perp}$ and $p_{\|}$are the electron momenta in the corresponding directions, $j=0,1,2, \ldots$.

We restrict our analysis to the case $j=0$ (bi-Maxwellian plasma). The generalization for $j \neq 0$ is straightforward (Tsai et al., 1981; Sazhin, 1989a). We also restrict ourselves to considering wave frequencies well above proton gyrofrequency so that the protons and other heavy ions could be considered as a neutralizing background. Finally we assume plasma to be weakly relativistic so that $p_{0 \perp(\|)} \ll m_{e} c$, where $m_{e}$ is the electron mass at rest, $c$ is the velocity of light. In view of all these assumptions and neglecting the contribution of higher-order terms, we can write the dispersion equation for parallel whistler-mode waves (cf. Sazhin, 1989a) as

$$
N^{2}=1-\frac{2 X}{r}\left[\mathscr{F}_{1 / 2,2}-\frac{\mathrm{d} \mathscr{F}_{3 / 2,2}}{\mathrm{~d} z}\left(A_{e}-1\right) N^{2}\right],
$$

where

$$
\begin{aligned}
& \mathscr{F}_{q, p} \equiv \mathscr{F}_{q, p}(z, a, b)=-i \int_{0}^{\infty} e^{i z t-\left[a t^{2} /(1-i t)\right]}(1-i t)^{-q}(1-i b t)^{-p} \mathrm{~d} t ; \quad(2.3) \\
& z=\frac{2(1-Y)}{r} ; \quad a=\frac{N^{2}}{r} ; \quad r=\frac{p_{0 \|}^{2}}{m_{e}^{2} c^{2}} ; \quad b=A_{e} ; \quad X=\Pi_{0}^{2} / \omega^{2} ; \\
& Y=\Omega_{0} / \omega ; \quad A_{e}=p_{0 \perp}^{2} / p_{0 \|}^{2} ;
\end{aligned}
$$


$\Pi_{0}, \Omega_{0}$, and $\omega$ are electron plasma frequency at rest, electron gyrofrequency at rest and wave frequency, respectively, $N$ is the wave refractive index.

We call the function $\mathscr{F}_{q, p}$ the generalized Shkarofsky function. It refers to the conventional Shkarofsky function (Robinson, 1986, 1987b)

$$
\widetilde{F}_{q}(z, a)=-i \int_{0}^{\infty} e^{i z t-\left[a t^{2} /(1-i t)\right]}(1-i t)^{-q} \mathrm{~d} t
$$

by the indentities

$$
\mathscr{F}_{q, 0}(z, a, b)=\mathscr{F}_{q, p}(z, a, 0)=\mathscr{F}_{q}(z, a) .
$$

Note that Robinson (1986) proposed another generalization of Shkarofsky function

$$
\mathscr{F}_{q, r}(z, a)=-i \int_{0}^{\infty} \mathrm{d} t \frac{(i t)^{r}}{(1-i t)^{q}} \exp \left[i z t-\frac{a t^{2}}{1-i t}\right],
$$

so that

$$
\mathscr{F}_{q, r}(z, a)=\frac{d^{r} \mathscr{F}_{q}(z, a)}{\mathrm{d} z^{r}} .
$$

The function $\mathscr{F}_{q, r}(z, a)$ is particularly important for the study of wave propagation in an isotropic plasma while the function $\mathscr{F}_{q, p}(z, a, b)$ is relevant to the analysis of wave propagation in an anisotropic plasma. New asymptotic analyses of the function $\mathscr{F}_{q, p}(z, a, b)$ are undertaken in the next section.

\section{Asymptotics for the Generalized Shkarofsky Function}

\subsection{Preliminary Remarks}

In nonrelativistic limit we can set $a \rightarrow \infty$ and reduce the function $\mathscr{F}_{q, p}$ to

$$
\mathscr{F}_{0}=-i \int_{0}^{\infty} e^{i z t-a t^{2}} \mathrm{~d} t=-\frac{1}{2 \sqrt{a}} Z\left(\frac{z}{2 \sqrt{a}}\right),
$$

where

$$
Z(\xi)=i \sqrt{\pi} \exp \left(-\xi^{2}\right)-2 \int_{0}^{\xi} \exp \left(-\xi^{2}+t^{2}\right) \mathrm{d} t
$$

The parameter $z$ is also large in the nonrelativistic case unless $Y$ is close to unity, while $\xi=z / 2 \sqrt{a}$ can in general be arbitrary. 
Nonrelativistic parallel whistler-mode propagation has been studied in detail by Sazhin (1983, 1986, 1989b).

In the limiting case $a \rightarrow \infty$ and $\xi=z / 2 \sqrt{a} \rightarrow-\infty$ but retaining the first order terms we simplify $\overline{\mathscr{F}}_{q, p}$ to

$$
\mathscr{F}_{q, p}=\frac{1}{z}+\frac{a}{z^{3}}\left(2-\frac{(q+b p) z}{a}\right) .
$$

Equation (3.3) is particularly convenient for the study of weakly relativistic effects on whistler-mode propagation (see, e.g., Jacquinot and Leloup, 1971; Sazhin, 1987a, b, 1989a).

In this paper we consider other asymptotic estimates of the function $\mathscr{F}_{q, p}$ as $a \rightarrow \infty$. We put $\mu=z / a$, and pay special attention to the limiting case $\mu \rightarrow 0$. All the parameters of the function $\mathscr{F}_{q, p}$ are assumed to be real $(z<0 ; a>0 ; p>0 ; q>0 ; b>0)$. Physically this assumption means that wave amplification or damping does not have influence on wave propagation.

\subsection{AN ALTERNATIVE FORM OF $\mathscr{F}_{q, p}$}

In this subsection we derive an asymptotic expansion of $\widetilde{\mathscr{F}}_{q, p}$ as $a \rightarrow \infty$, which is uniformly valid with respect to $\mu$ in a domain containing the point $\mu=0$. At the same moment we expand the results for small values of $\mu$.

Putting $t=i s$ we can write

$$
\mathscr{F}_{q, p}=\int_{0}^{-i \infty} \exp (\varphi(s)) f(s) \mathrm{d} s,
$$

where

$$
\begin{aligned}
& \varphi(s)=-z s+\frac{a s^{2}}{1+s}, \\
& f(s)=(1+s)^{-q}(1+b s)^{-p} .
\end{aligned}
$$

We assume that the branch cuts of $f(s)$ are taken along the real interval $(-\infty, 0)$. The path of integration is not restricted to the negative imaginary axis, but can be deformed according to our wishes.

We can rearrange the right-hand side of Equation (3.4) so that $\mathscr{F}_{q, p}$ is written as

$$
\mathscr{F}_{q, p}=e^{z-2 a} \int_{0}^{-i \infty} e^{a \psi(s)} f(s) \mathrm{d} s,
$$

where

$$
\psi(s)=(1-\mu)(s+1)+\frac{1}{s+1}
$$


Saddle points of $\psi$ are determined by the condition

$$
\frac{\mathrm{d} \psi(s)}{\mathrm{d} s}=(1-\mu)-\frac{1}{(s+1)^{2}}=0,
$$

which is satisfied for

$$
s=s_{ \pm} \equiv-1 \pm \frac{1}{\sqrt{1-\mu}} .
$$

In view of the future application of our analysis to whistler-mode waves $(Y>1)$ we consider $\mu \leq 0$. The analysis remains valid if $0<\mu<1$.

When $|\mu| \ll 1$ we have

$$
\begin{aligned}
& s_{+}=\frac{\mu}{2}+O\left(\mu^{2}\right), \\
& s_{-}=-2-\frac{\mu}{2}+O\left(\mu^{2}\right) ;
\end{aligned}
$$

$\psi(s)$ is real on the real axis and on the circle with the centre $s=-1$ and radius $1 / \sqrt{1-\mu}$. This circle passes through $s_{ \pm}$. Hence, $\psi(s)$ is real and decreasing along the contour shown in Figure 1. This saddle point contour is selected as a contour of integration.

We write $\mathscr{F}_{q, p}$ as

$$
\mathscr{F}_{q, p}=\mathscr{F}_{q, p}^{(1)}+\mathscr{F}_{q, p}^{(2)}+\mathscr{F}_{q, p}^{(3)},
$$

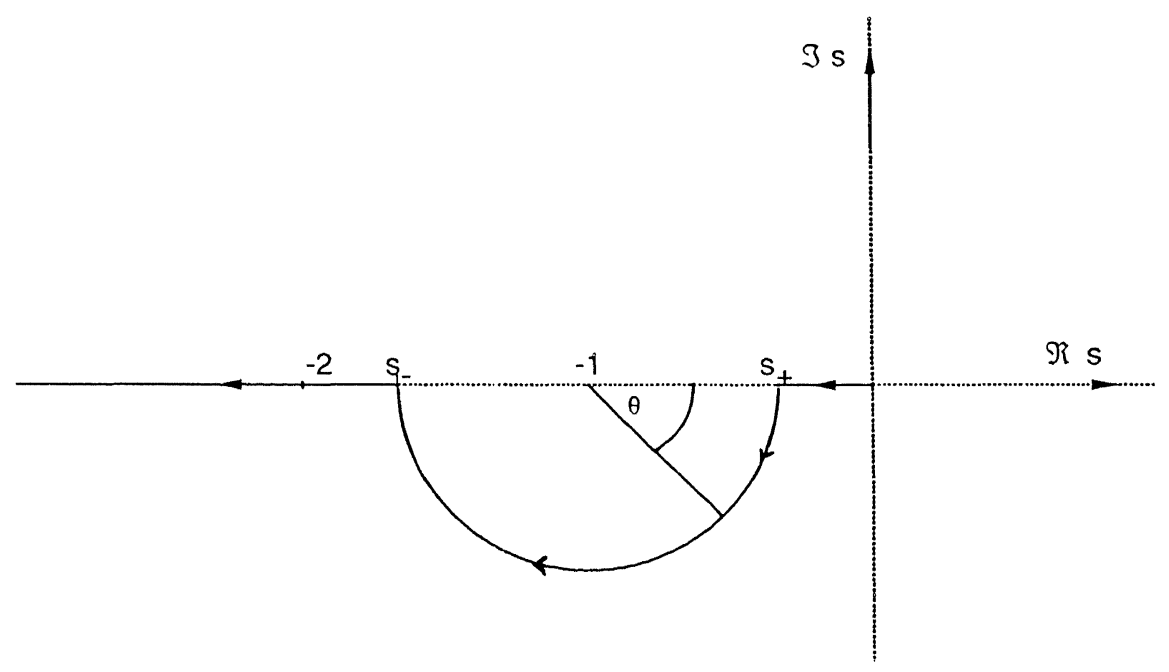

Fig. 1. The contour of integration in the complex $s$-plane. 
where

$$
\begin{aligned}
& \mathscr{F}_{q, p}^{(1)}=e^{z-2 a} \int_{0}^{s_{+}} e^{a \psi(s)} f(s) \mathrm{d} s, \\
& \mathscr{F}_{q, p}^{(2)}=e^{z-2 a} \int_{0}^{-\pi} e^{a \psi(s)} f(s) \frac{\mathrm{d} s}{\mathrm{~d} \theta} \mathrm{d} \theta, \\
& \mathscr{F}_{q, p}^{(3)}=e^{z-2 a} \int_{s_{-}}^{-\infty} e^{a \psi(s)} f(s) \mathrm{d} s .
\end{aligned}
$$

In (3.12) we put

$$
s=-1+\frac{e^{i \theta}}{\sqrt{1-\mu}}, \quad-\pi \leq \theta \leq 0 .
$$

The term $\mathscr{F}_{q, p}^{(1)}$ gives the dominant contribution to the real part of $\mathscr{F}_{q, p}$. The term $\mathscr{F}_{q, p}{ }^{(2)}$ contributes to the imaginary and real parts of $\mathscr{F}_{q, p}$, while the contribution of $\mathscr{F}_{q, p}{ }^{(2)}$ can be neglected when compared with that of $\mathscr{F}_{q, p}(1)$.

When $|\mu|$ is small the saddle point $s_{+}$is near the point $s=0$, the end point of integration of $\mathscr{F}_{q, p}$ and $\mathscr{F}_{q, p}^{(1)}$. The main contribution to the integral (3.5) comes from the point $s=0$; when $|\mu|$ is small also the influence of the saddle point $s_{+}$becomes important.

\subsection{ANALySis of $\mathscr{F}_{q, p}(1)$}

We transform

$$
\psi(s)-\psi\left(s_{+}\right)=x^{2},
$$

or

$$
x=\sqrt{\psi(s)-\psi\left(s_{+}\right)}
$$

$s=0$ corresponds to $x=x_{0}=\sqrt{\psi(0)-\psi\left(s_{+}\right)}=\sqrt{2-\mu-2 \sqrt{1-\mu}}, s=s_{+}$corresponds to $x=0$. The sign of $x$ and $x_{0}$ equals that of $\mu$. For small values of $|\mu|$ we have:

$$
x_{0}=\frac{1}{2} \mu+\frac{1}{8} \mu^{2}+O\left(\mu^{3}\right)
$$

This transformation enables us to write

$$
\mathscr{F}_{q, p}^{(1)}=-e^{-a x_{0}^{2}} \int_{0}^{x_{0}} e^{a x^{2}} g(x) \mathrm{d} x,
$$


where

$$
\begin{aligned}
& g(x)=f(s) \frac{\mathrm{d} s}{\mathrm{~d} x}, \\
& \frac{\mathrm{d} s}{\mathrm{~d} x}=\frac{2 x(1+s)^{2}}{(1-\mu)(1+s)^{2}-1},
\end{aligned}
$$

$g(x)$ can be Taylor-expanded at $x$ close to $x_{0}$ as

$$
g(x)=g\left(x_{0}\right)+g^{\prime}\left(x_{0}\right)\left(x-x_{0}\right)+\cdots,
$$

where

$$
\begin{aligned}
& g\left(x_{0}\right)=-\frac{2 x_{0}}{\mu}=-1-\frac{\mu}{4}+O\left(\mu^{2}\right), \\
& g^{\prime}\left(x_{0}\right)=-2 \frac{\mu^{2}+2 x_{0}^{2} \mu(q+p b)-4 x_{0}^{2}}{\mu^{3}}=1-q-p b+O(\mu) .
\end{aligned}
$$

If we substitute (3.17) into (3.14) and remembering the definition of $Z(\xi)$ (see Equation (3.2)) we obtain:

$$
\underset{\mathscr{F}_{q, p}}{(1)} \sim \frac{g\left(x_{0}\right)}{2 \sqrt{a}} \mathscr{R} Z\left(x_{0} \sqrt{a}\right)+\frac{g^{\prime}\left(x_{0}\right)}{4 a}\left[\mathscr{R} Z^{\prime}\left(x_{0} \sqrt{a}\right)+2 \exp \left(-a x_{0}^{2}\right)\right]
$$

as $a \rightarrow \infty$ with

$$
x_{0} \sqrt{a}=\xi\left[1+\frac{1}{4} \mu+\mathrm{O}\left(\mu^{2}\right)\right] .
$$

In view of (3.21) the term $\mathscr{R} Z\left(x_{0} \sqrt{a}\right)$ can be written as

$$
\mathscr{R} Z\left(x_{0} \sqrt{a}\right)=\mathscr{R} Z(\xi)+\frac{\xi^{2}}{2 \sqrt{a}} \mathscr{R} Z^{\prime}(\xi) .
$$

If we remember (3.18), (3.19), (3.21), and (3.22) and keep only the first-order terms with respect to $1 / \sqrt{a}$, we can simplify $(3.20)$ to

$$
\begin{aligned}
\mathscr{F}_{q, p}^{(1)} \sim \frac{1}{2 \sqrt{a}}\left\{-\mathscr{R} Z-\frac{\xi}{2 \sqrt{a}}\left(\mathscr{R} Z+\xi \mathscr{R} Z^{\prime}\right)+\right. \\
\left.\quad+\frac{1-q-p b}{2 \sqrt{a}}\left(\mathscr{R} Z^{\prime}+2 \exp \left(-\xi^{2}\right)\right)\right\} .
\end{aligned}
$$

This equation is more reliable for $\xi^{2} \geq 1$ than for $\xi^{2} \ll 1$. In the latter case the parameters $a$ and $z$ in (2.3) can no longer be considered as real, as the imaginary part of frequency $\omega$ is of the same order of magnitude or even larger than $\min \left(\omega, \Omega_{0}-\omega\right)$. Physically this means that the influence of wave damping or amplification on wave propagation can 
no longer be neglected (cf. the analysis of Sazhin (1983) of nonrelativistic whistler-mode waves at frequencies close to electron gyrofrequency)

3.4. ANALYSIS OF $\mathscr{F}_{q, p}^{(2)}$

For the semi-circular part of the contour shown in Figure 1 we have

$$
s+1=\frac{e^{i \theta}}{\sqrt{1-\mu}} .
$$

Equation (3.24) enables us to write

$$
\mathscr{F}_{q, p}^{(2)}=e^{-a x_{0}^{2}} \int_{0}^{-\pi} e^{-2 a \sqrt{1-\mu}(1-\cos \theta)} f(s) \frac{\mathrm{d} s}{\mathrm{~d} \theta} \mathrm{d} \theta .
$$

If we put $w=-\sin \frac{1}{2} \theta$, we can rewrite (3.22) as

$$
\mathscr{F}_{4, p}^{(2)}=e^{-a x_{0}^{2}} \int_{0}^{1} e^{-4 a \sqrt{1-\mu} w^{2}} \tilde{g}(w) \mathrm{d} w,
$$

where

$$
\begin{aligned}
& \tilde{g}(w)=f(s) \frac{\mathrm{d} s}{\mathrm{~d} \theta} \frac{\mathrm{d} \theta}{\mathrm{d} w}, \\
& \frac{\mathrm{d} s}{\mathrm{~d} \theta}=i(s+1), \\
& \frac{\mathrm{d} \theta}{\mathrm{d} w}=\frac{-2}{\sqrt{1-w^{2}}} .
\end{aligned}
$$

When $a x_{0}^{2} \gg 1$, the contributions from (3.26) are exponentially small, and not relevant for $\widetilde{F}_{q, p}$. In any case, the main contribution to the integral of (3.26) comes from the point $w=0$.

If we expand

we obtain

$$
\tilde{g}(w)=\sum_{k=0}^{\infty} c_{k} w^{k},
$$

$$
\begin{aligned}
\mathscr{F}_{q, p}^{(2)} \sim e^{-a x_{0}^{2}} & \sum_{k=0}^{\infty} c_{k} \int_{0}^{\infty} e^{-4 a \sqrt{1-\mu} w^{2}} w^{k} \mathrm{~d} w= \\
& =\frac{1}{2} e^{-a x_{0}^{2}} \sum_{k=0}^{\infty} c_{k} \Gamma\left(\frac{k+1}{2}\right)[4 a \sqrt{1-\mu}]^{-(k+1) / 2},
\end{aligned}
$$

for $a \rightarrow \infty$. 
The zeroth-order term $k=0$ in (3.28) gives an imaginary contribution. If we remember that

$$
c_{0}=\tilde{g}(0)=-2 i\left(1+s_{+}\right) f\left(s_{+}\right),
$$

we obtain using this term only

$$
\mathscr{F}_{q, p}^{(2)} \sim-\frac{i \sqrt{\pi} f\left(s_{+}\right)}{2 \sqrt{1-\mu} \sqrt{a \sqrt{1-\mu}}} e^{-a x_{i}^{2}},
$$

where $s_{+}$is defined by (3.7) and (3.8).

When $|\mu| \ll 1$ and if we remember (3.21) we can simplify Equation (3.29) to

$$
\underset{\mathscr{F}_{q, p}^{(2)}}{{ }^{(2)}} \sim-\frac{i \sqrt{\pi}}{2 \sqrt{a}}\left[1+\frac{\xi}{2 \sqrt{a}}\left(3-2 \xi^{2}-2 q-2 b p\right)\right] \exp \left(-\xi^{2}\right) .
$$

When deriving (3.30) we took into account that

$$
e^{-a x_{0}^{2}}=e^{-\xi^{2}}\left[1-\frac{\xi^{2} \mu}{2}\right]
$$

In zeroth-order approximation we have

$$
\mathscr{F}_{q, p}^{(2)}=-\frac{i}{2 \sqrt{a}} \mathscr{I} Z(\xi) .
$$

The term with $k=1$ in (3.28) gives a real correction to (3.20). Some algebraic manipulation yields

$$
c_{1}=\tilde{g}^{\prime}(0)=\frac{-4\left(1+s_{+}\right) f\left(s_{+}\right)}{1+b s_{+}}\left[(1-q)\left(1+b s_{+}\right)-p b\left(1+s_{+}\right)\right] .
$$

For small values of $|\mu|$ we have

$$
c_{1}=4(q+p b-1)+O(\mu) .
$$

A first-order approximation of the real part of $\mathscr{F}_{q, p}^{(2)}$ becomes

$$
\mathscr{R}_{q, p}^{(2)} \sim \frac{c_{1} e^{-\xi^{2}}}{8 a \sqrt{1-\mu}}
$$

when $a \rightarrow \infty$.

In view of (3.34) Equation (3.35) can be simplified to

$$
\mathscr{R}_{q, p}^{(2)} \sim \frac{q+p b-1}{2 a} e^{-\xi^{2}}
$$




\section{Propagation of the Waves}

If we collect the results (3.23) and (3.36) we have

$$
\begin{aligned}
\mathscr{R} \mathscr{F}_{q, p}= & \frac{1}{2 \sqrt{a}}\left[-\mathscr{R} Z-\frac{\xi}{2 \sqrt{a}}\left(\mathscr{R} Z+\xi \mathscr{R} Z^{\prime}\right)+\frac{1-q-p b}{2 \sqrt{a}} \mathscr{R} Z^{\prime}\right], \\
\mathscr{R} \frac{\mathrm{d} \mathscr{F}_{q, p}}{\mathrm{~d} z}= & \frac{1}{4 a}\left[-\mathscr{R} Z^{\prime}-\frac{1}{2 \sqrt{a}}\left(\mathscr{R} Z+\xi \mathscr{R} Z^{\prime}\right)-\right. \\
& \left.-\frac{\xi}{2 \sqrt{a}}\left(2 \mathscr{R} Z^{\prime}+\xi \mathscr{R} Z^{\prime \prime}\right)+\frac{1-q-p b}{2 \sqrt{a}} \mathscr{R} Z^{\prime \prime}\right] .
\end{aligned}
$$

In the limit $|\xi| \gg 1$ these expressions can be simplified to

$$
\begin{aligned}
& \mathscr{R} \mathscr{F}_{q, p} \sim \frac{1}{2 \sqrt{a}}\left[\frac{1}{\xi}+\frac{1}{2 \xi^{3}}\right], \\
& \Re \frac{\mathrm{d} \mathscr{F}_{q, p}}{\mathrm{~d} z} \sim-\frac{1}{4 a}\left[\frac{1}{\xi^{2}}+\frac{3}{2 \xi^{4}}\right] .
\end{aligned}
$$

When deriving (4.3)-(4.4) we took into account the inequality $|\xi| \ll 2 \sqrt{a}$ which follows from our assumption $|\mu| \ll 1$ and neglected the contribution of higher-order terms. In the limit $|\mu| \ll 1$ Equation (3.3) also reduces to (4.3). If we substitute (4.3)-(4.4) into the dispersion equation (2.2) we obtain the value of $N$ as a sum of wave refractive index in a cold plasma $N_{0}$ and nonrelativistic thermal corrections to $N_{0}$. These corrections have been extensively studied in our previous papers (see, e.g., Sazhin, 1986).

Let us now consider a special case $\xi=-0.924 \equiv-k$. For this value of $\xi$ we have $\mathscr{R} Z=-1 / \xi, \mathscr{R} Z^{\prime}=0, \mathscr{R} Z^{\prime \prime}=2 / \xi$ and

$$
\begin{aligned}
& \mathscr{R} \mathscr{F}_{q, p}=\frac{1}{2 \sqrt{a}}\left[-\frac{1}{k}+\frac{1}{2 \sqrt{a}}\right], \\
& \mathscr{R} \frac{\mathrm{d} \mathscr{F}_{q, p}}{\mathrm{~d} z}=\frac{1}{8 a \sqrt{a}}\left[2 k-\frac{3}{k}+\frac{2(q+b p)}{k}\right] .
\end{aligned}
$$

If we substitute (4.5)-(4.6) into Equation (2.2) we obtain

$$
N^{2}=N_{0}^{2}-\frac{X}{2 N^{2}}\left\{1-N \sqrt{r}\left(A_{e}-1\right)\left[2 k-\frac{3}{k}+\frac{3\left(1+4 A_{e}\right)}{3 k}\right]\right\} .
$$

If we, following Sazhin (1989a), neglect the contribution of the term proportional to $N \sqrt{r}$, then Equation (4.7) simplifies to 


$$
N^{2}=N_{0}^{2}-\frac{X}{2 N^{2}}
$$

This is exactly the result obtained by Sazhin (1989a). The coincidence of the results obtained by different methods can prove the validity of both approaches to the problem.

The expression (4.8) also follows from (4.7) in the case of whistler-mode propagation in an isotropic plasma $\left(A_{e}=1\right)$.

\section{Marginal Stability of the Waves}

The condition of marginal stability of the waves is equivalent to the condition that the imaginary part of the right-hand side of (2.2) is equal to zero. In view of (3.30) the latter condition can be written as

$$
\begin{aligned}
& {\left[Y+A_{e}(1-Y)\right]\left[1+\frac{\xi^{2}(Y-1)}{N^{2}}\right]+} \\
& \quad+\frac{Y-1}{N^{2}}\left\{2 A_{e}-1+\frac{(Y-1)\left(A_{e}-1\right)\left[A_{e}+\xi^{2}\left(1.5-2 A_{e}\right)\right]}{\xi^{2}}\right\}=0 .
\end{aligned}
$$

In nonrelativistic limit $(N \rightarrow \infty)$ Equation (5.1) is satisfied when

$$
A_{e}=\frac{Y}{Y-1}=A_{c r} .
$$

The waves are unstable when $A_{e}>A_{c r}$ and are damped when $A_{e}<A_{c r}$.

In a weakly relativistic limit we can expect that $A_{e}$ satisfying (5.1) should be close to $A_{c r}$ and so we can put $A_{e}=A_{c r}$ in the terms proportional to $N^{-2}$. As a result we can write the approximate solution of $(5.1)$ as

$$
A_{e}=\frac{Y}{Y-1}+\delta
$$

where

$$
\delta=\frac{Y-1+2 Y \xi^{-2}}{2(Y-1)\left[N^{2}+\xi^{2}(Y-1)\right]}>0 .
$$

As follows from (5.3) $A_{e}>A_{c r}$ which means that relativistic effects tend to stabilize propagating waves. This is consistent with the results of numerical analysis of Jacquinot and Leloup (1971).

Let us consider the values of the parameters used by Jacquinot and Leloup (1971) in their Figure 5: $j=0 ; \Pi_{0} / \Omega_{0}=1 ; A_{e}=2$; and $w_{\|}^{2} / c^{2}=0.03$; note that $\left\langle v_{\|}^{2}\right\rangle$, considered by Jacquinot and Leloup is equal to $0.5 w_{\|}^{2}$ in our notations. Based on our Equation (5.4) we calculated the frequency of marginal stability of whistler-mode waves 
$\left(\omega_{c r}\right)$ within non-relativistic and weakly relativistic approximations. In the nonrelativistic case $\omega_{c r}=0.5 \Omega_{0}$. This value of $\omega_{c r}$ coincides with the corresponding value of $\omega_{c r}$ obtained by Jacquinot and Leloup (1971). For the weakly relativistic case we obtained $\omega_{c r} \approx 0.45 \Omega_{0}$. This value of $\omega_{c r}$ is slightly larger than $\omega_{c r}$ obtained by Jacquinot and Leloup (1971), as from their Figure 5 it follows that $\omega_{c r} \approx 0.42 \Omega_{0}$. However, for the considered values of the parameters we have $|\mu|=2(Y-1) / N^{2} \approx 0.4$. So, we can expect that the contribution of the terms proportional to $\mu^{2}$ which we neglected in our analysis are not negligible. This could be the cause of the slight discrepancy between our results and those of Jacquinot and Leloup (1971), although we have a qualitative agreement between them.

Expression (5.4) can be further simplified when

$$
1 \ll \xi^{2}(Y-1) \ll N^{2}
$$

and reduced to

$$
\delta=1 / 2 N^{2} .
$$

In another limiting case

$$
1 \ll N^{2} \ll \xi^{2}(Y-1)
$$

it reduces to

$$
\delta=1 /\left((Y-1) \xi^{2}\right) .
$$

The condition (5.5) is relevant to a very hot plasma, while the condition (5.7) is satisfied for a nearly cold plasma.

As follows from (5.4), $\delta$ may be significant when whistlers are propagating through a rarefied ( $N^{2}$ is not too large) and hot ( $\xi^{2}$ is not too large) plasma. An example of such a plasma was considered when we compared our results with those of Jacquinot and Leloup (1971). The ratio $\Pi_{0} / \Omega_{0}$ of the order of unity was often observed in the magnetosphere of the Earth in the region outside the plasmasphere (see, e.g., Curtis, 1978). However, the value of $w_{\|}^{2} / c^{2}=0.03$ is very extreme rather than typical value of this parameter in this region (Hess, 1968; Bahnsen et al., 1985). More realistically we can set $w_{\|}^{2} / c^{2}=0.004$ (which corresponds to electrons with energies of the order of $1 \mathrm{keV}$ ). If we assume as it was done above, $\Pi_{0}=\Omega_{0}, A_{e}=2$, we obtain $\delta \approx 0.01$ and the weakly relativistic value of $\omega_{c r}$ equal to $\approx 0.49 \Omega_{0}$. It seems realistic to distinguish experimentally this value of $\omega_{c r}$ from the non-relativistic value $\omega_{c r}=0.5 \Omega_{0}$, although serious problems may arise due to the uncertainty of the determination of other parameters of the magnetospheric plasma.

When applying our equations to practical analysis of the waves in the magnetospheric and/or astrophysical plasmas we should remember the main restriction of our theory: $|\mu|=2(Y-1) / N^{2} \ll 1$. In particular, our theory would not be valid for a very rarefied plasma when $N^{2} \approx 1$. Also, our theory is not valid when $\omega$ is very close to $\Omega_{0}$, i.e., when the increment of instability or decrement of damping of the waves is of the order of, or above $\left|\mathscr{R} \omega-\Omega_{0}\right|$. 


\section{Conclusions}

On the basis of asymptotic analysis of a generalized Shkarofsky function we have considered the problem of parallel whistler-mode propagation and marginal stability in a weakly relativistic plasma in the limit of a large wave refractive index $N$. In the low temperature limit relativistic effects do not influence on wave propagation provided $N \gg 1$. For $\xi=-0.924$ we have generalized the expression for $N$ obtained earlier by Sazhin (1989a). In contrast to Sazhin (1989a), it is pointed out that this expression depends on $A_{e}$, although this dependence is not strong when $N \sqrt{r} \ll 1$. Relativistic effects result in the increase of the value of anisotropy for which the waves are unstable. It is pointed out that the degree of this increase could be observed experimentally in the conditions of the magnetosphere of the Earth.

\section{Acknowledgements}

One of the authors (S.S.) would like to thank the Center for Mathematics and Computer Science (Amsterdam) as well as SERC for financial support of this project. He is also grateful to the staff of this Center for the hospitality during his stay in Amsterdam in February 1989.

\section{References}

Anderson, R. A.: 1983, Rev. Geophys. 21, 474.

Bahnsen, A., Jespersen, M., Neubert, T., Canu, P., Borg, H., and Frandsen, P. E.: 1985, Ann. Geophys. 3, 19.

Bornatici, M. and Ruffina, U.: 1989, in Contributed Papers of the 16th European Conference on Controlled Fusion and Plasma Physics, Venice, March 13-17, part 4, p. 1381-1384.

Carpenter, D. L.: 1988, Rev. Geophys. 26, 535.

Curtis, S. A.: 1978, J. Geophys. Res. 83, 3841.

Helliwell, R. A.: 1988, Rev. Geophys. 26, 551.

Hess, W. N.: 1968, The Radiation Belt and Magnetosphere, Blaisdell, Waltham, Massachusetts.

Inan, U. S.: 1987, Rev. Geophys. 25, 588.

Jacquinot, J. and Leloup, C.: 1971, Phys. Fluids 14, 2440.

Robinson, P. A.: 1986, J. Math. Phys. 27, 1206.

Robinson, P. A.: 1987a, J. Plasma Phys. 37, 149.

Robinson, P. A.: 1987b, J. Math. Phys. 28, 1203.

Sazhin, S. S.: 1982, Natural Radio Emissions in the Earth's Magnetosphere, Nauka, Moscow (in Russian).

Sazhin, S. S.: 1983, J. Plasma Phys. 29, 217.

Sazhin, S. S.: 1986, Ann. Geophys. 4, 155.

Sazhin, S. S.: 1987a, J. Plasma Phys. 37, 209.

Sazhin, S. S.: 1987b, J. Plasma Phys. 38, 301.

Sazhin, S. S.: 1989a, Phys. Scripta 40, 114.

Sazhin, S. S.: 1989b, Planetary Space Sci. 37, 311.

Scarf, F. L., Gurnett, D. A., Kurth, W. S., Coroniti, F. V., Kennel, C. F., and Poynter, R. L.: 1987, J. Geophys. Res. 92, 15, 217.

Shkarofsky, I. P.: 1966, Phys. Fluids 9, 561.

Shkarofsky, I. P.: 1986, J. Plasma Phys. 35, 319.

Trubnikov, B. A.: 1959, in M. A. Leontovich (ed.), Plasma Physics and the Problem of Controlled Thermonuclea Reactions, Pergamon Press, London.

Tsai, S. T., Wu, C. S., Wang, Y. D., and Kang, S. W.: 1981, Phys. Fluids 24, 2186.

Tsang, K. T.: 1984, Phys Fluids 27, 1659. 